\section{Evaluation of Initial Treatment of Trochanteric Fractures using Gamma Nails ORIGINAL}

\section{Abstract}

Background: Trochanteric fractures are extra-joint fractures, account for $55 \%$ of femoral proximal fractures. They often happen in the elderly with increased frequency due to age. Treatment experiences at 7A Military Hospital (Ho Chi Minh city, Viet Nam) showed increased trochanteric fracture cases. To accurately assess treatment effectiveness and select proper treatment methods, the study of evaluation of initial treatment of trochanteric fractures using gamma nails and made the conclusions of indications and applied techniques was carried out.

Methods and findings: Twenty-three patients diagnosed with traumatic trochanteric fractures and surgically treated with Gamma nails were monitored and evaluated for bone fusion, bone union, range of motion, limb shortening, and functionalrehabilitation according to Merle D'Aubigné scale.

Nineteen patients (82.6\%) achieved proper bone union withthe femoral neck-shaft angle of $125^{\circ}-130^{\circ}$, four patients (17.4\%) got slightly deviated bone union.There were $20 / 23$ cases (87.0\%) achieved "Good" and "Very good" functional rehabilitation, scored over 15 points according to Merle D'aubigne Index; $3 / 23$ cases (13.0\%) got "Average" recovery. Hip range of motion in 20 cases (87.0\%) achieved "Very good" (normal movement) or "Good" (over 10\% movement limitation) outcome, three cases had "Average" movement (13.0\%). Limb shortening of below $1 \mathrm{~cm}$ took place in 14 patients $(60.9 \%), 1-2 \mathrm{~cm}$ in 5 patients (21.7\%) and over $3 \mathrm{~cm}$ in 4 patients $(17.4 \%)$.

Conclusions: The surgical treatment oftrochanteric fractures using gamma nails yielded positive outcomes.
Quang-Tri Lê', Minh Hoang Nguyen ${ }^{1}$

1 Department of Orthopedics, 7A Military Hospital. 466 Nguyen Trai Street, Ward 8, District 5, Ho Chi Minh City, 72706, Viet Nam.

\section{Contact information:}

\section{Quang-Tri Lê.}

Address: Director of 7A Military Hospital. Head of Department of Orthopedics, Head of Department of High-Tech Diagnostics, 466 Nguyen Trai Street, Ward 8, District 5, Ho Chi Minh City, 72706, Viet Nam. Tel: (+084)0839241868.

”- lqtri@ntt.edu.vn tsbstri@yahoo.com 


\section{Introduction}

Trochanteric fractures accounted for $55 \%$ of proximal femoral fractures, by definition, include fractures in the area from the femoral neck to $5 \mathrm{~cm}$ below the lesser trochanter. Trochanteric region has a thin outside bone structure with the spongy bone inside, quickly become frail with age, especially in case of osteoporosis. Trochanteric fractures usually happen in the elderly and with a clear female dominance[1].

Surgery treatments for trochanteric fractures have become the recommended treatment, while functional and conservative treatments are only applicable in rare cases with non-displace fractures or strictly contra-indication to surgery. Treatments with internal fixation devices, including intramedullary and extramedullary fixation devices, are widely used with advantages and disadvantages [2]

Gamma nail treatment is a classic intramedullary fixation device that combines a sliding hip screw with a primary nail fit into the intra medulla cavity. Treatments with Gamma nails has been widely used internationally with several advantages reported, such as increasing stability at the fracture site, advancing healing, shortening operation time, and allowing early rehabilitation [3].

Cho Ray Hospital (one the top hospital in Vietnam) from June 2003 to December 2005 applied bone fusion using Gamma nails for surgical treatment of 42 trochanteric fracture patients with orthopedic scanning using the C-Arm machine. The results were $100 \%$ bone union, 10\% deviation, 10\% limb shortening, and functional rehabilitation outcomes were $55 \%$ very good, $35 \%$ good, and $10 \%$ average [4].

Recently 7A Military Hospital has applied bone fusion with orthopedic surgical table and C-Arm scanning and achieved initial positive results. To accurately assess treatment effectiveness and select proper treatment methods, the study of "Evaluation of initial treatment of trochanteric fractures using gamma nails at 7A Military Hospital" was carried out with the goals of:
- Evaluation of initial treatment of trochanteric fractures using gamma nails at the 7A Military Hospital.

- Drawing of conclusions of indications and applied techniques.

\section{Methods}

Research targets: Twenty three patients diagnosed with traumatic trochanteric fractures and surgically treated with Gamma nails. Bone fusion, bone union, range of motion, limb shortening, and functional rehabilitation, according to Merle D'Aubignéscale were monitored and evaluated [3].

Location: Department of Orthopedics, 7A Military Hospital, Ho Chi Minh City, Viet Nam

Time: June 2014 to June 2019.

Criteria for selection: include adult patients with closed traumatic trochanteric fractures of $A 1$ and A3 types (AO classification) and surgically treated at 7A Military Hospital.

Criteria for rejection: include patients with pathologic or progressive infection trochanteric fractures, having pre-fracture paralysis, or health conditions unsuitable for surgical operation.

\section{Research Methods}

The study has cross-sectional description design.

Samples were intentional chosen based on the above selection and rejection criteria.

Bone fusion procedures: The patient lied on orthopedic table. An incision was made from the greater trochanter apex to expose the fracture site fully. Drill to make a hole at MCL site and on the marrow canal. Then the nail was inserted, apparatus installed and screwing.

Postoperative treatments: The patients were rested with the leg fixed on Braun splint. Preventive antibiotics were used before, during, and after operations together with painkillers, anti-edema agents, and taking good care of the wound. Cardiopulmonary resuscitation and chronic disease treatment were applied when needed. 
Postoperative exercises included muscular static stretching of wounded limb, joint movements in bed with staff assistance, and crutch-assisted active exercises without pressing on the wounded limb during the first month. Then the joint range of motion was checked-up; clinical and sub-clinical bone radiography was done to plan for the next stage of exercises. After that, a periodic check-up was done once in three months, and the followup exercises were designed based on examination results.

Researched parameters were ages and sexes; cause of fractures; anatomical, pathological traits of injuries; surgical indications; surgery time; short term and long term outcomes; conditions of surgical scars, bone union, and functional rehabilitation.

\section{Data analysis}

The data were collected using one unified form. Data sources were historical records, interviews, clinical examinations, surgical operations, postoperative exercises, discharge assessment, and records of fusion apparatus carrying time. The data were analyzed using common medical statistics.

\section{Result assessment}

Short term outcomes were assessed based on Larson-Bosman criteria.

Within less than 9 months after operation, the surgical wound conditions, the fracture site conditions, and the bone deviation via radiography immediately, 3 months and 6 months after the operation were evaluated.

Long term outcomes were assessed based on criteria of Merle D'Aubigné [5].

1. Very good

- Radiography: stable union, no deviation, femoral neck-shaft angle of $120^{\circ}-130^{\circ}$

- Functional rehabilitation: 17-18 points on Merle D'Aubigné scale

- No limb shortening or shortening $<1 \mathrm{~cm}$

- No complication
2. Good

- Radiography: union, slight deviation, neck-shaft angle of $120^{\circ}-130^{\circ}$

- Functional rehabilitation: 15-16 points on Merle D'Aubigné scale

- Limb shortening of 1-2 cm

3. Average

- Radiography: union, large deviation, neck-shaft angle of $<110^{\circ}$

- Functional rehabilitation: 13-14 points on Merle D'Aubigné scale

- Limb shortening of 2-3 cm or shallow infection

4. Poor

- Radiography: non-union, screw loosening or other complications

- Functional rehabilitation: 13-14 points on Merle D'Aubigné scale

- Limb shortening of $>3 \mathrm{~cm}$ or deep infection, prolonged fistula inflammation

\section{Results}

Out of 23 researched patients, there were 15 patients (65.2\%) aged less than 60 years, and eight patients were over 60 years of age (34.8\%). Males patients were 8 cases, accounted for 34.8\%, and female patients were 15 cases, accounted for $65.2 \%$. Male/female ratio was nearly $1 / 2$.

Regarding the causes of fractures, the majority was domestic accidents with 11 cases (47.8\%), in which 4/11 cases were less than 60 years old (36.4\%), and $7 / 11$ cases were over 60 years old (63.6\%). Traffic accidents caused 10/23 cases of fractures, accounted for $43.5 \%$, in which $6 / 10$ cases $(60 \%)$ were less than 60 years old, and the remaining $4 / 10$ cases (40\%) were over 60 years old. Work accidents caused the two remaining cases accounted for $8.7 \%$, dividing equally to 1 male and one female patients. 
Based on A/O system, the 23 cases of trochanteric fractures were divided into basicervical fractures (4 patients, 17.4\%), lesser trochanteric fractures (12 patients, 52.2\%), and subtrochanteric fractures (7 patients, 30.4\%). There were no intertrochanteric fracture case in this study.

Table 1 described the A/O classification [4] per ages of 23 studied cases. The A1 and A3 were indicated for surgery, and $A 3$ cases took a more significant percentage (19/23 patients, 82.6\%).

Time from trauma till operation varies from less than five days to more than ten days. Most of the patients had surgery within less than five days of injury (21 patients, 91.4\%). There was one patient who had operated eight days after trauma (4.3\%) and one after 12 days (4.3\%). The average time from fracture to operation was 4.8 days. Most patients were treated within the first week since fracture $(91.4 \%)$.

Postoperation hospitalization time varied from 7 days to more than 15 days. Most of the cases (19 patients, $82.7 \%$ ) stayed less than ten days, three patients (13.0\%) stayed 10-15 days, and one patient stayed 18 days (4.3\%). The average hospitalization period was 8.9 days.

The shaft adjustment results after bone fusion were presented in Table 2 . The femoral neck-shaft of $120^{0}-130^{\circ}$ and non-deviated fracture sites were achieved in 19/23 patients (82.6\%). The Gamma screws were positioned correctly in the neck axis in all patients. Femoral neck-shaft of only $110^{\circ}$-below $120^{\circ}$ and fractures deviation by one bone shell took place in $4 / 23$ patients (17.4\%).

After 9-12 months following up, nineteen patients $(82.6 \%)$ had proper union with the neck-shaft angle of $125^{0}-130^{\circ}$, four patients (17.4\%) had slightly deviated union.

The walking rehabilitation results were showned in Table 3. There were 20/23 patients (87.0\%) achieved "Good" and "Very Good" results, and only $3 / 23$ patients (13.0\%) had "Average" outcome based on Merle D'aubigne scale.
Table 1. A/O classification per ages.

\begin{tabular}{l|c|c|c|c|c|c|}
\multicolumn{7}{|c|}{ A/O Classification } \\
\hline \multirow{2}{*}{ Group } & \multicolumn{5}{|c|}{ A1 } & \multicolumn{3}{c|}{ A3 } \\
\cline { 2 - 8 } & A1.1 & A1.2 & A1.3 & A3.1 & A3.2 & A3.3 \\
\cline { 2 - 8 } & $\%$ & $\%$ & $\%$ & $\%$ & $\%$ & $\%$ \\
\hline Years & & & & & & \\
\hline \multirow{2}{*}{$<60$} & 13.0 & 0 & 4.3 & 8.8 & 17.4 & 21.7 \\
\hline \multirow{2}{*}{60} & $(3 / 23)$ & 0 & $(1 / 23)$ & $(2 / 23)$ & $(4 / 23)$ & $(5 / 23)$ \\
& 0 & 0 & 0 & 8.8 & 13.0 & 13.0 \\
$(2 / 23$ & $(3 / 23)$ & $(3 / 23)$ \\
\hline
\end{tabular}

Table 2. Bone fusion results $(n=23)$.

\begin{tabular}{|c|c|c|}
\hline \multicolumn{2}{|c|}{ Shaft adjustment results } & $\%$ \\
\hline \multicolumn{3}{|l|}{ Neck-shaft angle } \\
\hline $1200-1300$ & $82.6(19 / 23)$ & 87.50 \\
\hline 1100-below 1200 & $17.4(4 / 23)$ & 62.50 \\
\hline Below 1100 & 0 & 68.75 \\
\hline \multicolumn{3}{|l|}{ Fracture condition } \\
\hline Straight with the shaft & $82.6(19 / 23)$ & 87.50 \\
\hline Deviation by one shell & $17.4(4 / 23)$ & 62.50 \\
\hline \multicolumn{3}{|l|}{ Fracture condition } \\
\hline Correct & $100(23 / 23)$ & 87.50 \\
\hline Incorrect & 0 & 62.50 \\
\hline
\end{tabular}

Table 3. Walkingrehabilitation $(n=23)$.

\begin{tabular}{|l|c|c|}
\hline \multirow{2}{*}{ Shaft adjustment results } & \multicolumn{2}{|c|}{ Patients } \\
\cline { 2 - 3 } & $\%$ & Outcomes \\
\hline $\begin{array}{l}\text { Normal walking without pain } \\
\begin{array}{l}\text { Limping, pain with excessive } \\
\text { walking }\end{array}\end{array}$ & $26.1(14 / 23)$ & Very good \\
\hline $\begin{array}{l}\text { Pain when walking, requiring } \\
\text { crutch }\end{array}$ & $13.0(3 / 23)$ & Good \\
\hline
\end{tabular}

Hip range motion recovery assessment was presented in Table 4. Twenty patients (87.0\%) got "Very good" and "Good" results in hip range of motion (normal or reduced movement of $\geq 10 \%$ ), and three patients (13.0\%) got "Average" results.

Limb shortening results was shown in Table 5 . There was less than $10 \mathrm{~cm}$ in 14 patients (60.9\%), $1-2 \mathrm{~cm}$ in 5 patients (21.7\%) and $\geq 3 \mathrm{~cm}$ in 4 patients (17.4\%). There was no patient has the limb shorter than $4 \mathrm{~cm}$. 
Table 4. Recovery of hip range of motion $(n=23)$.

\begin{tabular}{|l|c|c|}
\hline \multicolumn{1}{|c|}{ Outcomes } & Hip range of motion & Percentage \\
\hline Very good & Normal & $60.9(14 / 23)$ \\
\hline Good & Reduce of 10\%-30\% & $26.1(6 / 23)$ \\
\hline Average & Reduce of 30\%-50\% & $13.0(3 / 23)$ \\
\hline
\end{tabular}

Table 5. Limb shortening results $(n=23)$.

\begin{tabular}{l|c|c|}
\multicolumn{1}{|c|}{ Outcomes } & Limb shortening & $\%$ \\
\hline Very good & No shortening or $<1 \mathrm{~cm}$ & $60.9(14 / 23)$ \\
\hline Good & $1-2 \mathrm{~cm}$ & $21.7(5 / 23)$ \\
\hline Average & $3-4 \mathrm{~cm}$ & $17.4(4 / 23)$ \\
\hline Poor & $>4 \mathrm{~cm}$ & 0
\end{tabular}

\section{Discussion}

Trochanteric fractures arethe common fractures in the elderly $[3,7,8]$, and they are accompanied by chronic diseases such as osteoporosis, hypertension, and diabetes [9-11, 12], and can cause various complications [3, 12-14], for example,compressive lesion, respiratory and urinary tract inflammation due to prolonged bed confinement, asthenia, and limb shortening. Consequently, early diagnosis and surgical intervention are essential for complication prevention. Movement rehabilitation is for convenience in daily life. Bone fusion using Gamma nails is complicated surgery requires adequate facilities for anesthesia, sterilization, and advanced equipment.

Through the study, we observed that bone fusion using Gamma nails for trochanteric fractures has many advantages, for examples:

- Appropriate and affordable fusion devices suitable forthe actual conditions of both healthcare facilities and patients.

- The Gamma nails, thanks to their structure, generate compression on both fraction surfaces, which increases the stability of fracture sites and promotes bone union in the initial stage. Patients can sit up early, can conveniently taking self-care, and soon participa- te in post-operative rehabilitative exercises, which preventsystemic and localized illnesses caused by prolonged bed confinements.

- Cancellous screws and Gamma nails together strongly hold femoral neck and shaft in place and increase fracture stability during motion.

Also, patient physical health should be promoted. Osteoporosis medication such as Rocaltrol, Canxicorbier, or Miacanxic should be administered continuously two or three months after operation since osteoporosis in elder patients may cause screw loosening and secondary deviation of neck-shaft angle.

\section{Conclusion}

Treatment outcomes in this study showed that $16 / 19$ patients $(84.21 \%)$ got $120^{0}-130^{\circ}$ of femoral neck-shaft angle and non-deviated fracture site. All patients had gamma nails positioned correctly in the neck axis. Neck-shaft angle of $110^{\circ}-<120^{\circ}$ and deviation of one bone shell in 3/19 patients (15.79\%). Proper bone union, neck-shaft angle of $125^{0}-130^{0}$ were achieved in 14 patients (82.35\%), and three patients (17.65\%) had slightly deviated union. Amongst the aged under 60 group, 14/17 patients (82.35\%) got "very good" and "good" functional rehabilitation based on Merle D'aubigne scale.

$A 1$ and $A 3$ fractures (A/O classification) were indicated for surgery. Bone fusion using Gamma nails is complicated and delicate surgery, which requires advancedequipment; however, the 7A Military Hospital can afford such conditions to improve treatment efficiency.

\section{Declaration}

\section{Scientific Responsibility Statement}

The authors declare that they are responsible for the article's scientific content including study design, data collection, analysis and interpretation, 
writing, some of the main line, or all of the preparation and scientific review of the contents and approval of the final version of the article.

\section{Animal and human rights statement}

All procedures performed in this study were in accordance with the ethical standards of the institutional and/or national research committee and with the 1964 Helsinki declaration and its later amendments or comparable ethical standards.

\section{Funding}

None.

\section{Conflict of interest}

None of the authors received any type of financial support that could be considered potential conflict of interest regarding the manuscript or its submission.

\section{References}

1. Maville JA, Huerta CG. Health promotion in nursing. 3rd ed. USA: Delmar; 2013.

2. Han L, Liu J-J, Hu Y-G, et al. Controlled study on Gamma nail and proximal femoral locking plate for unstable intertrochanteric femoral fractures with broken lateral wall. Sci Rep. 2018; 8(1):11114. doi:10.1038/s41598-018-28898-6

3. Adam P. Treatment of recent trochanteric fracture in adults. OrthopTraumatolSurg Res. 2014; 100(1):S75-S83. doi:10.1016/j. otsr.2013.11.007

4. Le VT. Evaluation of trochanteric fractures treatment using Gamma nails at Cho Ray Hospital. Ho Chi Minh Orthopedics Association. 2007 (7). (In Vietnamese)
5. Biau DJ, Brand RA. Robert Merle d'Aubigne, 1900-1989. ClinOrthopRelat Res. 2009; 467(1):2-6.

6. Vo TP. Physical health of aged over 65 patients and orpthopedic surgery. J Med News. 2004; 9(2):81-83. (In Vietnamese)

7. Lustosa LP, Bastos EO. (2009). Proximal fracture of the femur on the elderly: what's the best treatment?. ActaOrtopédicaBrasileira. 2009; 17(5):309-312

8. Ricardo M. Treatment of Intertrochanteric Hip Fractures in Elderly High Risk Patients with External Fixation. OrthopRheumatol. 2016 Oct; 3(2):1-3

9. Ghoneem HF, Zayda Al, El Sayed AFA. Treatment of intertrochanteric fracture femur by llizarov fixator in elderly patients. Menoufia Med J. 2018; 31(4):1293-1298.

10. Gulcelik NE, Bayraktar M, Caglar O, Alpaslan M, Karakaya J. Mortality after hip fracture in diabetic patients. ExpClinEndocrinol Diabetes. 2011; 119(07):414-418.

11. Li Y, Lin J, Cai S, Yan L, Pan Y, Yao X, Zhuang H, Wang P, Zeng Y. (2016). Influence of bone mineral density and hip geometry on the different types of hip fracture. Bosnian J Basic Med. 2016; 16(1):35-8.

12. Lin JC-F, Liang W-M. Mortality and complications after hip fracture among elderly patients undergoing hemodialysis. BMC Nephrol. 2015; 16.

13. Baumgarten $M$, Margolis DJ, Orwig DL, Shardell MD, Hawkes WG, Langenberg $P$ et al. Pressure ulcers in elderly patients with hip fracture across the continuum of care. J Am Geriatr Soc. 2009; 57(5):863-870.

14. Yamaguchi $R$, Jono $O$, Hamasaki $A$, Sakamoto $A$, Tatsumoto $T$, Kita $\mathrm{M}$ et al. Early Operation for Femoral Trochanteric Fracture in Elderly: Cause of Delayed Operation. OrthopTraumatol. 2012; 61(2):265-269.
Publish in International Archives of Medicine

International Archives of Medicine is an open access journal publishing articles encompassing all aspects of medical science and clinical practice. IAM is considered a megajournal with independent sections on all areas of medicine. IAM is a really international journal with authors and board members from all around the world. 